\title{
Preface for the Special Issue of Hybrid Manufacturing
}

\author{
Guest Editors: Haedo Jeong, and Won-Shik Chu ${ }^{2}$ \\ 1 School of Mechanical Engineering, Pusan National University, 2, Busandaehak-ro 63beon-gil, Geumjeong-gu, Busan, 46241, South Korea \\ 2 Institute of Advanced Machines and Design, Seoul National University, 1, Gwanak-ro, Gwanak-gu, Seoul, 08826, South Korea \\ \# Corresponding Author / Email: hdjeong@pusan.ac.kr, TEL: +82-51-510-2463, FAX: +82-51-518-8442
}

The immergence of a paradigm sparks a prominent growth within a specific area or the time period. The keyword "hybrid" has been flooding diverse aspects within our economy and society, giving rise to hybrid cars, hybrid clubs, hybrid cameras, hybrid apps, hybrid bikes, hybrid bonds, and more. The word "hybrid" typically means the integration of two or more different elements into a whole. More generally, it means the combination of different elements, a method with which something new-like a market or a field-with increased added values can be created. In other words, it can be said that the concept of hybrid focuses on the convergence or the fusion of different functions.

The paradigm of hybrid is also actively being implemented and applied in manufacturing and processing. This is done by introducing new or different manufacturing methods into the traditional manufacturing schemes via hybridization to increase the productivity or the product quality. In the micro/nano scale manufacturing and processing, many researchers are trying to combine the different manufacturing processes not only enhance the productivity or the quality of the product, but also to fabricate a new product using multi-materials with function, for example. Still the dominant manufacturing processes are based on traditional manufacturing processes, many other nontraditional manufacturing are investigated and hybridized.

Many different definitions and classifications still exist since the issues of process timing and environment, features of geometry, and capable sizes for processing are not easy to compare. But the goal of hybrid manufacturing is removing the disadvantageous aspects out of the whole process, including, but not limited to, additional delivery, transport, delay in the process, energy consumption, increase of cost, etc. of hybrid manufacturing process.

Although the solid definition of hybrid manufacturing is not established, the future trend of hybrid manufacturing will include higher dimension of feature, multiple materials and functions, composites, ultra precision, rapid production, etc.

The papers in this special issue discusses the recent research trend in the fields of forging, polishing, surface texturing, analysis using laser, welding, milling, etc. in the facets of energy efficiency, effectiveness, performance enhancement, and manufacturing. Below are the topics in this special issue:
- Energy-Efficient Optimization of Forging Process

- Effect of Glycine on Copper CMP

- Electrically-Assisted Indentation

- Selective Electro-Thermal Growth of Nanowire

- Hot-Cold Complex Forging Technology

- Gold Current Collecting Layer

- Laser Induced Breakdown Spectroscopy Analysis

- Green-Environmental Dry Adhesive

- Laser-ARC Hybrid Welding

- Laser Assisted Milling Device

We would like to express our sincere appreciation to Prof. Chong Nam $\mathrm{Chu}$, the chair of International Symposium on Hybrid Manufacturing and president of Next Generation Hybrid Manufacturing Consortium at SNU for his efforts in organizing the symposium and special issue. We also thank all of the authors who provided their efforts to the articles in the special issue. We are also grateful to the members of Next Generation Hybrid Manufacturing Consortium at SNU and Hybrid Manufacturing Consortium at Korean Society for Precision Engineering (KSPE) for providing multiple reviews for the articles. We really appreciate their precious time and effort on the review. Lastly, we would like to thank Prof. Sung-Hoon Ahn (SNU, Korea), Editor-in-chief of IJPEM-GT, Prof. David Dornfeld (University of California, Berkeley, USA), Prof. Friedrich Prinz, (Stanford University, USA), Prof. Yoshimi Takeuchi (Chubu University, Japan) and Prof. Frank Vollertsen (BIAS - Institute of Applied Beam Technology, Germany), Co-Editors-in-Chief of IJPEMGT, and Prof. Choon-Man Lee, the president of KSPE, for giving us the opportunity to publish this special issue. 